\title{
Erratum 2: Difference between two species of emu hides a test for lepton flavour violation
}

\author{
Christopher G. Lester and Benjamin H. Brunt \\ University of Cambridge, Department of Physics, Cavendish Laboratory, \\ JJ Thomson Avenue, Cambridge, CB3 OHE, U.K. \\ E-mail: lester@hep.phy.cam.ac.uk, brunt@hep.phy.cam.ac.uk
}

ERratum TO: JHEP03(2017)149

ABSTRACT: In the original published version of this paper, the cross sections which were reported for the supersymmetric straw-models used in this paper were too large. This was a consequence of the authors' failure to notice that smuon widths were not being re-computed by MADGRAPH when model parameters were changed. This erratum contains replacements for the tables and figures which change as a result of using the correct smuon cross sections. Because the originally reported cross sections were overestimates, the corrected sensitivity is less than was originally claimed. Nonetheless, the analysis remains viable.

KEYWORDS: Supersymmetry Phenomenology

ARXIV EPRINT: 1612.02697 


\begin{tabular}{|lcrr|}
\hline Label on plots & $\begin{array}{c}\left(m_{\tilde{\mu}}, m_{\tilde{\chi}_{1}^{0}}\right) \\
\mathrm{GeV}\end{array}$ & $\lambda_{231}^{\prime}$ & $\begin{array}{c}\sigma_{\mathrm{RPV}} \\
\mathrm{fb}\end{array}$ \\
\hline RPV_50_500 & $(500,50)$ & 1.0 & 130 \\
RPV_150_1000 & $(1000,150)$ & 1.0 & 10 \\
RPV_50_500_0p2 & $(500,50)$ & 0.2 & 84 \\
\hline
\end{tabular}

Table 1. The example RPV SUSY models used in this document.

During the writing of a doctoral thesis [1] a bug was found in the way the authors of [2] had computed smuon widths in MADGRAPH. This resulted in overestimation of the signal cross sections for each of the three models used to illustrate viability of the search technique proposed in [2]. This erratum seeks to correct that mistake. Note that since the replacement figures are drawn (with permission) from [1]: (i) they are shown for a reference integrated luminosity $100 \mathrm{fb}^{-1}$ rather than the $20 \mathrm{fb}^{-1}$ assumed in [2], and (ii) the third model point in table 1 of this erratum has $\lambda_{231}^{\prime}=0.2$ rather than the 0.5 assumed in [2]. Neither of these changes is materially important to this erratum, whose sole objective is to place into the public domain a self-consistent set of error-free results for some integrated luminosity and for some straw models. The captions of the tables and figure provided in this erratum are written as if being dropped into the document which is being corrected.

- Table 1 of this erratum replaces the table 1 of [2].

- Figure 1 of this erratum replaces figure 1 of [2]. Note that the change of variable from $m_{T}(\mu)$ to $m_{T}(e)+m_{T}(\mu)$ is already explained in [3].

- Figure 2 of this erratum replaces figure 2 of [2].

- No replacement is needed for figure 3 of [2].

- The information which would be needed to produce a perfect replacement for figure 4 of [2] has sadly been lost. In the absence of that data, the most closely related guide is provided by figure 4 of [4] which (importantly) uses the correct $m_{T}(e)+m_{T}(\mu)$ variable, but which (regrettably) assumes only $20 \mathrm{fb}^{-1}$ of data and the old incorrect signal cross sections. Nonetheless, the cut values which the latter figure shows are not strongly sensitive to integrated luminosity, so little change is expected.

- Figure 3 of this erratum replaces figure 5 of [2].

- Figure 4 of this erratum replaces figure 6 of [2]. 


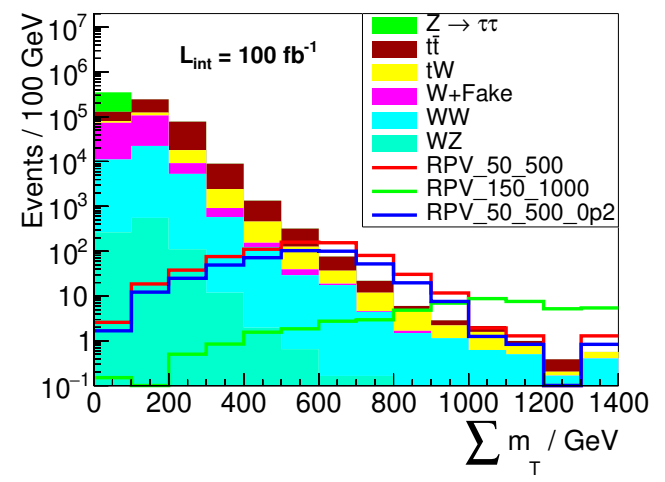

(a) $m_{T}(e)+m_{T}(\mu)$ in $e^{+} \mu^{-}$events.

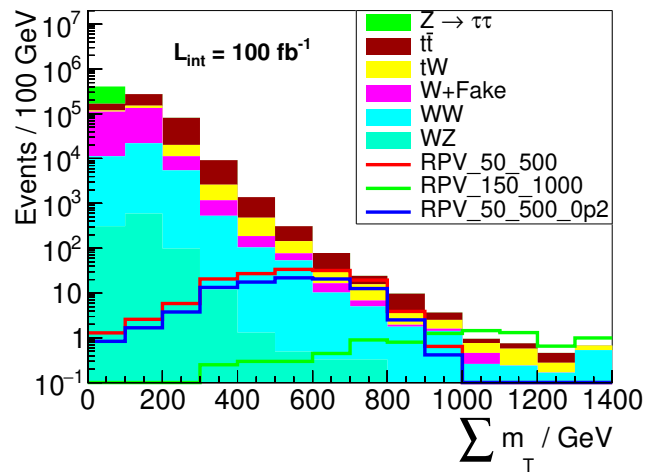

(b) $m_{T}(e)+m_{T}(\mu)$ in $e^{-} \mu^{+}$events.

Figure 1. The expected distributions of $m_{T}(e)+m_{T}(\mu)$ in events with OSDF leptons $\left(e^{+} \mu^{-}\right.$and $e^{-} \mu^{+}$in (a) and (b) respectively).

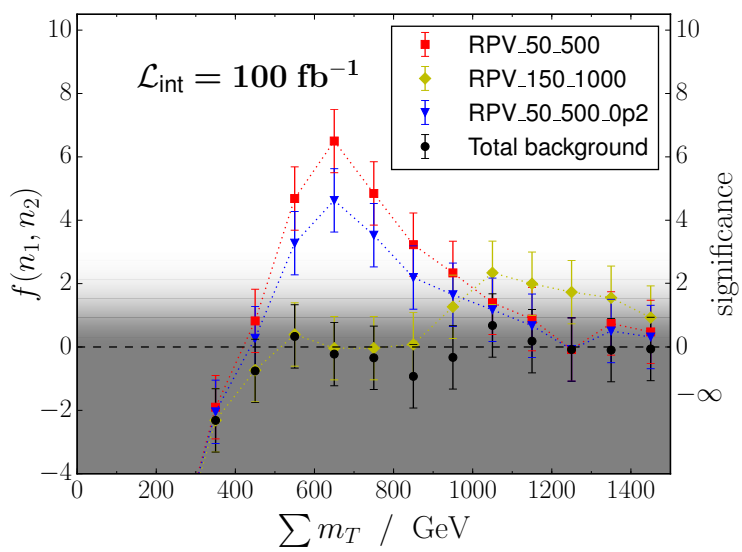

Figure 2. The left-hand axis shows the median value of the statistic $f\left(n_{1}, n_{2}\right)$ in each $50 \mathrm{GeV}$ bin of $m_{T}(e)+m_{T}(\mu)$, while the right-hand axis shows the mapping of these values to 'sigmas significance' using the blue line of figure 3 (of [2]). The black points show background alone, and the coloured points show the sum of the background with each of the example signals. Error bars indicate the $50 \pm 34$ th percentile values of $f$, i.e. the $\pm 1 \sigma$ deviations from the median. The shaded region indicates the null hypothesis of $f\left(n_{1}, n_{2}\right) \leq 0$ and unit variance upwards. The dotted lines connecting points are given as a guide to the eye. 


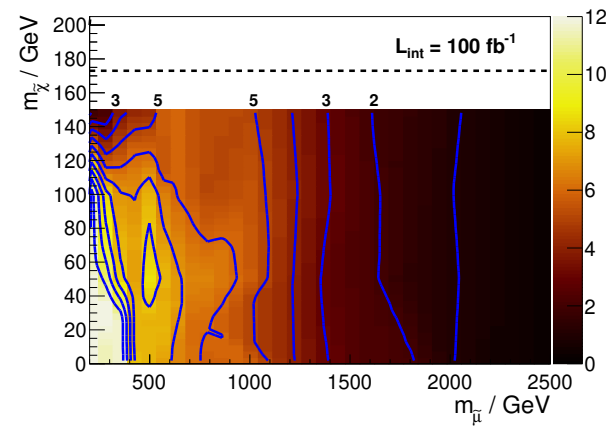

Figure 3. The median value of $f$ (in effect the expected sensitivity of the method) for the grid of $\lambda_{231}^{\prime}=1$ signal models. Contour lines show integer values of sensitivity.

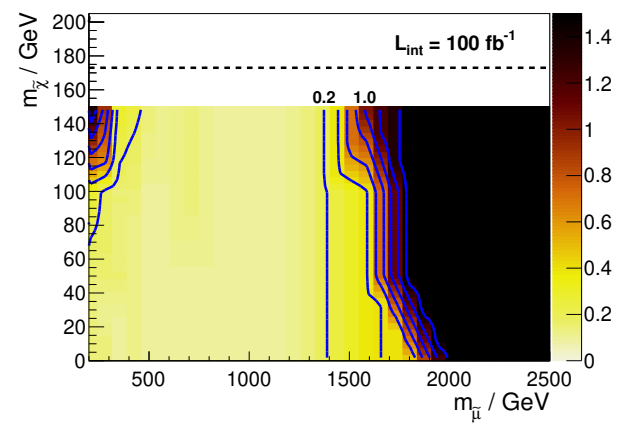

Figure 4. The minimal value in each model of the $\lambda_{231}^{\prime}$ coupling for which a sensitivity of $f \geq 2$ is achieved. Contour lines are drawn at intervals of 0.2 .

Open Access. This article is distributed under the terms of the Creative Commons Attribution License (CC-BY 4.0), which permits any use, distribution and reproduction in any medium, provided the original author(s) and source are credited.

\section{References}

[1] B.H. Brunt, Searches for new physics with the ATLAS experiment, CERN-THESIS-2018-422 (2018) [https://doi.org/10.17863/CAM.24188].

[2] C.G. Lester and B.H. Brunt, Difference between two species of emu hides a test for lepton flavour violation, JHEP 03 (2017) 149 [arXiv: 1612.02697v4].

[3] C.G. Lester and B.H. Brunt, Erratum to: Difference between two species of emu hides a test for lepton flavour violation, JHEP 08 (2017) 069.

[4] C.G. Lester and B.H. Brunt, Difference between two species of emu hides a test for lepton flavour violation, arXiv:1612.02697v5. 